\title{
Ressources et limites d'un langage de la vengeance dans les réactions populaires aux attentats de Madrid (2004) et Paris (2015)
}

Resources and limits of a retaliatory language in the popular reactions to the attacks in Madrid (2004) and Paris (2015)

Recursos y límites de un lenguaje de venganza en las reacciones populares frente a los atentados de Madrid (2004) y Paris (2015)

\section{Myriam Cremer et Rolf Kailuweit}

\section{OpenEdition}

Journals

Édition électronique

URL : https://journals.openedition.org/mots/23922

DOI : $10.4000 /$ mots. 23922

ISSN : 1960-6001

Éditeur

ENS Éditions

Édition imprimée

Date de publication : 5 novembre 2018

Pagination : 117-135

ISBN : 979-10-362-0082-3

ISSN : 0243-6450

\section{Référence électronique}

Myriam Cremer et Rolf Kailuweit, « Ressources et limites d'un langage de la vengeance dans les réactions populaires aux attentats de Madrid (2004) et Paris (2015) », Mots. Les langages du politique [En ligne], 118 | 2018, mis en ligne le 05 novembre 2020, consulté le 23 avril 2022. URL : http:// journals.openedition.org/mots/23922 ; DOI : https://doi.org/10.4000/mots.23922 


\section{Ressources et limites d'un langage de la vengeance dans les réactions populaires aux attentats de Madrid (2004) et Paris (2015)}

Les attentats islamistes qui ont frappé Madrid le 11 mars $2004^{1}$ et Paris en janvier et novembre $2015^{2}$ ont profondément touché à ce que William Connolly (2005, p. 11) appelle le « registre d'existence viscéral ». L'expérience d'une telle souffrance met en branle des processus affectifs qui libèrent des émotions et des passions profondes pouvant donner naissance à un désir de vengeance. Cependant, dans les sociétés occidentales, fondées sur le principe d'étatisation du droit pénal ainsi que sur les valeurs chrétiennes, l'action vengeresse constitue un tabou. Comment, alors, exprimer son désir de vengeance si celuici est jugé moralement condamnable?

La recherche n'a pas encore prêté d'attention à l'émergence d'un langage de la vengeance en réponse aux attaques terroristes, ce qui ne paraît guère surprenant dans la mesure où ce dernier constitue une catégorie minoritaire des réactions populaires à ces attentats dans les sociétés occidentales. En effet, Gérôme Truc (2016, p. 236 et suiv.) a mis en évidence que les valeurs les plus citées dans un corpus de presque 59000 messages numériques consécutifs aux attentats du 11 mars 2004 à Madrid sont la paix (14500 occurrences)3, l'amour4 (plus de

1. Le 11 mars 2004, dix bombes explosèrent dans quatre trains de banlieue, dans les gares Santa Eugenia, El Pozo et Madrid-Atocha, causant la mort de 192 personnes et en blessant près de 1900 autres. Bien que le gouvernement d'Aznar ait immédiatement accusé l'organisation nationaliste basque ETA, les preuves indiquèrent rapidement la responsabilité d'Al-Qaïda (Reinares, 2014).

2. Le 7,8 et 9 janvier 2015, une série d'attaques islamistes à Paris et dans sa proche banlieue visèrent la rédaction du journal satirique Charlie Hebdo, une policière et un supermarché cacher, faisant au total 17 morts et plusieurs dizaines de blessés. Le 13 novembre 2015,130 personnes furent tuées, et plus de 400 blessées, dans des attentats revendiqués par l'État islamique visant des bars et restaurants du $10^{\mathrm{e}}$ et $11^{\mathrm{e}}$ arrondissements ainsi que la salle de spectacle du Bataclan et le Stade de France.

3. Cependant, il faut noter qu'environ 1800 de ces occurrences font partie des formules telles que «Reposez en paix» (Truc, 2016, p. 237).

4. Dans ce contexte, G. Truc (2016, p. 249) met en relation l'amour invoqué dans les messages

Heinrich-Heine Universität Düsseldorf myriam.cremer@hhu.de Heinrich-Heine Universität Düsseldorf rolf.kailuweit@hhu.de 
2 200) et la vie (plus de 2 500), tandis que la vengeance reste marginale. Il est donc naturel que les chercheurs se concentrent en premier lieu sur l'exploration des manifestations de la solidarité (Truc, 2006, 2011, 2016) ou bien sur la construction de la mémoire (Ortiz García, 2011, 2013). Cependant, d’un point de vue psychologique (McCullough, 2008), le désir de vengeance n'est pas moins intéressant, étant donné qu'il est considéré comme une réaction naturelle après un événement traumatisant. Et c'est pourquoi, dans une perspective qualitative, les ressources rhétoriques qui sont mobilisées pour sublimer le désir de vengeance représentent un domaine de recherche du plus haut intérêt.

Notre contribution propose une approche sémiolinguistique afin de mettre en perspective les enjeux soulevés par la mise en lumière d'un langage de la vengeance. Les résultats initiaux de notre recherche en cours se fondent, concernant l'attentat de Madrid, sur un ensemble de 70000 documents provenant de l'Archivo del Duel05. Dans le détail, la documentation comprend 2532 photographies, 495 objets et 6436 messages ou dessins sur papier qui ont été retirés des gares lorsque les mémoriaux populaires ont été remplacés par un dispositif informatique ${ }^{6}$, grâce auquel ont été enregistrés, de juin 2004 à mars 2005, 58732 messages supplémentaires intégrés à l'archive. Pour ce qui est des attentats de 2015 à Paris, nous nous appuierons en premier lieu sur un corpus composé de 7700 documents numérisés par les Archives de Paris qui, peu après les attaques du 13 novembre 2015, ont commencé à collecter les messages déposés sur les sites des attentats7. Il n'y eut pas de collecte équivalente après les attentats des 7, 8 et 9 janvier 2015. Lors de deux séjours de recherche aux Archives de Paris en mai 2017, nous avons cependant eu accès à 3858 documents provenant des fonds privés, documentant les mémoriaux surgis place de la République et devant le siège de Charlie Hebdo. Ces matériaux ont en outre pu être enrichis par 875 documents récoltés par les Archives de Rennes à la suite des commémorations des attentats de $2015^{8}$, que nous avons consultés en août 2017.

Étant donné la nature hétérogène de ces matériaux9 et la place marginale qu'y occupent les messages exprimant un désir de vengeance, l'étude livrée

post-attentats avec l'idée de l'agapè (amour inconditionnel, universel), telle que la conceptualise Luc Boltanski (1990).

5. Fonds d'archives créé par une équipe de chercheurs du CSIC (Consejo Superior de Investigaciones Científicas), aujourd'hui conservé au Musée national du chemin de fer espagnol, à Madrid.

6. Après le démantèlement et l'archivage des mémoriaux populaires le 9 juin 2004 , des bornes informatiques ont été placées dans les gares d'Atocha, d’El Pozo et de Santa Eugenia où les passants pouvaient écrire un message de condoléances. Il était également possible de le faire directement sur le site web mascercanos.com (Truc, 2011).

7. Àl'exception du Stade de France.

8. 650 messages du corpus concernent les attentats de janvier (série 2250W) et 225 messages ceux de novembre (série $2265 \mathrm{~W}$ ).

9. Papiers, cartons, images, dessins, objets tridimensionnels, notes manuscrites ou imprimées, messages électroniques ainsi que des photos contextualisant les messages textuels. 
ici sera essentiellement qualitative. Nous ne nous interdirons pas pour autant, sur la base d'études approfondies que nous avons pu mener sur certains de ces message ${ }^{10}$, de donner parfois quelques indicateurs de tendance concernant telle ou telle stratégie rhétorique. Il importe néanmoins de garder à l'esprit que les conditions d'écriture des messages et le contexte communicatif ont une influence surl'émergence et la documentation des désirs de vengeance qui sont socialement tabous ${ }^{11}$. On sait que les mémoriaux populaires sont souvent entretenus par des « gardiens » (Gensburger, 2017; Truc, Bazin, 2019), qui suppriment les feuilles volantes comportant des remarques inappropriées, de sorte que la fréquence avec laquelle ces remarques se produisent effectivement est difficile à calculer. Lorsque les messages sont rédigés sur ordinateur, les scrupules peuvent diminuer, à moins que, comme dans le cas des bornes informatiques installées à Madrid, l'acte d'écriture lui-même, souvent effectué en groupe, reste soumis à un certain contrôle social (Truc, 2011). Aussi est-il peu surprenant que sur un total de presque 59000 messages numériques collectés par ce dispositif il n'y en ait que 80 dans lesquels apparaissent les termes vengar ("venger») ou venganza ("vengeance»), dont 26 seulement expriment un désir de vengeance. Il s'ensuit que les quantifications sont tout au plus possibles par rapport à des situations de communication particulières et bien documentées. Nous opterons donc, ici, pour une approche essentiellement qualitative fondée sur des analyses linguistiques des rôles sémantiques (Kailuweit, Hummel, 2004; Rappaport Hovav, Levin, 2015) et sur la fonction pragmatique des actes de langages (Austin, 1975), ainsi que, pour ce qui est des messages visuels ou texto-visuels, sur la sémiologie de Roland Barthes (1964a, 2014).

Notre recherche se structure autour de l'antagonisme opposant d'un côté la raison et les conventions morales rejetant toute volonté de vengeance, et de l'autre le désir de restaurer par un acte vengeur la stabilité ébranlée par un attentat terroriste. Nous partirons de l'hypothèse principale que, en l'absence d'une capacité d'agir effective, le langage peut représenter un instrument compensateur, en tant qu'il permet par le truchement de redéfinitions ou de projections de s'approprier une capacité d'agir. Dans cette perspective, nous nous focaliserons sur la construction de l'agency (capacité d'agir effective ou imaginée) dans la zone conflictuelle entre vouloir-faire (désir de vengeance) et nepas-pouvoir-faire (défini par les normes sociales, morales et juridiques) ${ }^{12}$. Sur

10. Voir Quintana et Kailuweit (2017) pour une analyse détaillée des messages numériques archivés à Madrid sous l'aspect pragma-linguistique de l'intensification.

11. Dans ce contexte, Cristina Sánchez-Carretero (2011b, p. 257) part de l'hypothèse que l'amour est sur les feuilles, tandis que la haine s'inscrit sur les murs, hypothèse qui ne s'est confirmée qu'en partie. Même sur les murs, les messages xénophobes et les cris de vengeance ne sont pas très fréquents.

12. Nous retenons la distinction faite par Algirdas Julien Greimas (1976) entre les modalités extrinsèques telles que le devoir- ou bien le pouvoir-faire (déterminées par les normes et les valeurs socioculturelles) et les modalités intrinsèques telles que le savoir-faire et en premier lieu le vouloir-faire (déterminées par nos passions affectives). 
ce plan, nous suivons l'approche sociologique de Cornelia Helfferich (2012, p. 13 et suiv.) pour qui la notion d'agency permet de comprendre comment un individu, un groupe ou un artefact est doté d'une certaine capacité d'agir, ou bien à qui ou à quoi celle-ci est attribuée. L'attribution d'agency reste une construction subjective qui ne correspond pas nécessairement à une capacité d'agir effective. Elle peut même être fictive en imaginant un acte vengeur (agency imaginée au sens de Pamela Steen, 2012).

Après quelques réflexions théoriques concernant les mémoriaux populaires post-attentats, l'expression d'un désir de vengeance et ses enjeux en termes d'agency, nous en viendrons dans un second temps à l'étude des ressources linguistiques d'un langage de la vengeance au travers d'une analyse comparative des données recueillies après les attentats de Madrid en 2004 et de Paris en 2015.

\section{Exprimer un désir de vengeance dans les mémoriaux populaires post-attentats}

La dimension collective des attaques terroristes apparait d'autant plus évidente quand une ville est attaquée en son cœur, causant la mort de nombreux civils. Ainsi, peu après les attentats de Madrid en 2004 et de Paris en 2015, un grand nombre de citoyens se sont réunis sur les lieux des attentats ou les places centrales de la ville (telles que la place de la République à Paris) pour rendre hommage aux victimes. Beaucoup d'entre eux ont exprimé leur solidarité en allumant des bougies ou en déposant des fleurs, des peluches, des photos, des dessins ou encore des messages écrits plus ou moins élaborés et de longueurs diverses. Se sont ainsi formés ce que Peter J. Margry et Cristina Sánchez-Carretero (2011a) englobent sous le concept de «mémoriaux populaires» (grassroots memorials). Ces mémoriaux, qui ne sont pas propres aux attentats $^{13}$, mettent en lumière l'impact collectif de l'événement en transformant le deuil privé en deuil collectif par des rites et pratiques de commémoration publique et en créant des espaces éphémères d'action collective (Doss, 2006 ; Fraenkel, 2011 ; Margry, Sánchez-Carretero, 2011b ; Sánchez-Carretero, 2011b ; Truc, 2016). En cela, ils permettent d'exprimer des protestations en incluant, dans leur capacité de transformation sociale, des messages politiques (Santino, 2011, p. 105). Si les travaux en sciences sociales sur ces mémoriaux constituent un utile point de départ pour notre étude sur le langage de la vengeance, ils mettent toutefois peu l'accent sur les aspects linguistiques et sémiotiques.

13. Outre les attentats terroristes, les événements pouvant conduire à l'apparition de tels mémoriaux sont divers : catastrophes aériennes, accidents de circulation, tueries de masse et fusillades scolaires, morts de célébrités, etc. 
Ce sont en premier lieu des travaux en philosophie (Elster, 1990 ; Erman, 2012 ; Hénaff, 2000)14, en psychologie (McCullough, 2008) et en droit (Rosenbaum, 2013) qui s’intéressent à la problématique phénoménologique de la vengeance ainsi qu'à l'évaluation normative de celle-ci. Comme le montrent ces études, la plupart des réflexions sur la notion de vengeance partent de la loi du talion provenant du Code de Hammurabi, ancien texte juridique babylonien, d'où vient la formule bien connue, œil pour œil, dent pour dent ${ }^{15}$. Dans cette optique, la vengeance s'inscrit dans un système de parité tentant a priori de limiter d'éventuels excès relatifs à l'acte vengeur. Cependant, dès qu'il s'agit de rendre compte de la vengeance en tant que réaction civile, de telles définitions s'avèrent limitées. L'étude de Maartje Elshout et ses coauteurs (2015) a repéré un réseau complexe de notions associées au concept de vengeance tel qu'il est compris par la société civile. Ils ont mis en évidence que le désir de vengeance reste souvent inextricablement lié au désir de retourner à un équilibre de puissance et de se faire justice soi-même en infligeant une souffrance à ceux qui nous ont fait souffrir. Dès lors qu'il s'agit de venger une mort violente, il est souvent question d'infliger aux responsables le maximum de souffrances.

Étudier les ressources et limites d'un langage de la vengeance implique de faire une distinction nette entre l'acte de vengeance (faire) et le désir de vengeance (vouloir-faire), ce dernier relevant de l'imaginaire per se sans revêtir le caractère illicite lié au passage à l'action. Puisque la réalisation du désir de vengeance est socialement inacceptable, les frontières entre dire et agir changent. L'acte de langage lui-même, dans une certaine mesure, prend la place de l'acte de vengeance. Notre hypothèse est donc que le sujet cherche à traduire son désir de vengeance dans un acte sémiolinguistique performatif. En adoptant le concept de la performativité tel qu'il a été introduit par John L. Austin (1975), nous focaliserons notre attention sur les pratiques sémiotiques, en particulier les actes d'écriture (Fraenkel, 2007), qui, en mobilisant un effort cognitif et linguistique plus ou moins faible, pourraient générer dans le contexte postattentats des effets perlocutoires remarquables.

Un des enjeux centraux liés à l'appropriation d'une capacité d'agir réside dans le fait que l'attribution d'agency engage une responsabilité tant morale que sociale (Helfferich, 2012 ; Lucius-Hoene, 2012). Étant donné que la revendication de responsabilité par rapport à une action vengeresse s'avère problématique, nous nous interrogerons sur les stratégies linguistiques que le sujet de l'énonciation peut développer afin de réduire sa responsabilité. L'agency peut en effet être modulée par le choix des prédicats et des rôles sémantiques, ainsi que par d'autres stratégies linguistiques au niveau de l'énonciation (la diathèse, les constructions impersonnelles, etc.), ou bien par des marqueurs

14. Il ne s'agit ici bien sûr que d'une sélection fournie à titre d'exemple.

15. Nous retrouvons cette formule dans des textes religieux tels que l'Ancien Testament (Ex. 21, 24-5). 
d'évidentialité (par exemple le conditionnel) et par des verbes épistémiques au niveau des actes de langage. La délégation de responsabilité par projection de l'action vengeresse dans un espace sémiologique imaginaire (Greimas, Fontanille, 1991) constitue également une stratégie possible. Ainsi accorderons-nous une attention particulière à la place du mythe, tel que l'entend R. Barthes (2014 [1957]) qui, en s’appuyant sur la distinction entre dénotation et connotation de Louis Hjelmslev (1961 [1943]), montre que des signes linguistiques peuvent, par le biais d'une signification seconde, souvent cachée (connotation), dépasser le sens dénotatif des informations, et être ainsi porteurs de mythes.

\section{Le langage de la vengeance après les attentats de Madrid et Paris : une analyse comparative}

Dans notre corpus, nous avons repéré des exemples ${ }^{16}$ qui montrent très clairement que le sujet éprouvant un désir de vengeance après un attentat se trouve affecté par des charges modales contradictoires et, d'un point de vue social et moral, incompatibles : le désir de vengeance en tant que vouloir-venger (motivé par la souffrance et la colère) ou bien devoir-venger (souvent ressenti par les proches des victimes) se trouvant confronté à un ne-pas-pouvoir-venger, ou même ne-pas-devoir-venger. Les exemples suivants sont révélateurs de cette ambivalence axiologique résultant du hiatus entre le « cœur», lieu symbolique de nos activités émotionnelles, «cri[ant] vengeance», et la «raison» déterminée, dans nos sociétés occidentales, par les instances socioculturelles et morales appelant aux valeurs chrétiennes :

Le cœur crie vengeance, mais la raison appelle à l'apaisement et au pardon, - rose blanche pour la paix - (Archives de Rennes, 2265W1[40])

Solo Mi Razón Y Mi Dios, en estos momentos, de ODIO, ASCO, IRA REPUGNANCIA... Me hace no hacer con vosotros lo que HABEIS HECHO CON NOSOTROS. [Seuls ma raison et mon Dieu, dans ces moments de haine, de dégoût, de colère et de répugnance... m'empêchent de faire avec vous ce que vous avez fait avec nous.] (Archivo del Duelo, DP-0295)

Ce qui est intéressant ici, c'est le fait que l'interférence de ces dispositifs modaux conflictuels semble influer sur la modulation de l'agency. Dans le premier exemple, c'est par le biais des personnifications que le rôle de l'agent est extériorisé et attribué à deux instances contradictoires. Le «cœur» en tant que force intrinsèque entre donc en conflit avec la «raison » déterminée par des forces extrinsèques telles que les normes sociales. Cependant, il reste à savoir comment le conflit entre ces deux instances se terminera, même si la

16. Les citations empruntées aux corpus sont laissées dans leur état initial incluant toutes les fautes d'orthographe ou de frappe, fréquentes dans les messages. 
suite laisse supposer que la raison prévaudra. Dans le deuxième exemple, ce n'est pas seulement à la raison, mais aussi à Dieu - et ainsi à une instance morale supérieure - que le rôle d'agent est attribué, et cela de sorte que les deux agents empêchent le sujet de l'énonciation d'exécuter la vengeance. Dans l'exemple qui suit, le sujet de l'énonciation met en œuvre une autre stratégie. En avouant avoir des désirs de vengeance et en demandant pardon à une autorité morale supérieure, il renonce, bien que de manière implicite, à une capacité d'agir :

Espero que Dios sepa perdonarme, por mi Ira y mis deseos de venganza que aun perduran. [J'espère que Dieu saura me pardonner, pour ma colère et mes désirs de vengeance qui perdurent encore.] (Archivo del Duelo, EP, mascercanos.com, 10 juin 2004)

Le dernier exemple de ce type montre que le degré d'implication dans l'attentat et le sentiment individuel d'en avoir été ou non victime constituent un facteur important lorsqu'il s'agit de justifier rhétoriquement le désir de vengeance. Cependant, l'emploi du conditionnel réduit l'attribution de l'agency. Il n'est pas précisé si le sujet de l'énonciation cèdera à la pression morale en renonçant à son désir ou non.

Fui victima del atentado, megustaria pedir venganza aunque por lo visto no esta bien visto. [J'ai été victime de l'attaque, je voudrais demander vengeance bien qu'apparemment ce ne soit pas bien vu.] (Archivo del Duelo, EP, Atocha, 16 octobre 2004)

Ces exemples mettent en évidence que le combat intérieur entre les charges modales contradictoires débouche souvent sur le renoncement à la vengeance au profit des normes morales. Cependant, le langage met à disposition de nombreuses stratégies alternatives pour pouvoir exprimer le désir de vengeance sans pour autant enfreindre le canon des valeurs humanistes. Par la suite, il sera donc question de montrer comment le sujet de l'énonciation au niveau de l'énoncé, ou bien l'énonciateur à travers l'acte de langage, peuvent s'approprier une certaine capacité d'agir.

Les observations menées sur les corpus révèlent que le verbe venger est presque toujours employé sur le mode «venger quelqu'un», par exemple : «Je veux les venger. Je veux venger Mon Amie Marion partie trop tôt» (Archives de Paris, 3904W4[4]). L'acte terroriste en tant que tel ("venger quelque chose») apparait comme relégué au second plan derrière ceux qui en ont été directement victimes. Une analyse lexicale de nos données a montré en outre que beaucoup de messages manifestent un désir de vengeance sans pour autant recourir aux termes venger ou vengeance. Conformément aux observations de M. Elshout et ses coauteurs (2015), le désir de vengeance s'exprime régulièrement par une volonté de faire, ou voir, souffrir : «Les barbares méritent de souffrir et avoir les mêmes peines atroces qu'ont subi les victimes» (Archives de Paris, 
3904 W55[71]) ; "Il faudrait les faire souffrir pour tout le mal qu'ils ont causé» (Archives de Paris, 3904W5[205]); "espero que todos los terroristas sufran el mismo dolor que las familias afectadas, se lo merecen » [j'espère que tous les terroristes endureront les mêmes souffrances que les familles touchées, ils le méritent] (Archivo del Duelo, EP, Atocha, 20 septembre 2004). Une autre stratégie consiste à exiger la mort en tant que souffrance ultime : «Les gens qui ont fait ça devraient tous mourir pareil » (Archives de Paris, 3904W005[227]); «Ils seront anéantis quand nous les exterminerons» (Archives de Paris, 3904W49[88]); « rayer du monde tous ces putain de terroristes qui n'ont pas lieu d'être ici sur notre terre » (Archives de Paris, 3904W7[89]). Dans les réactions aux attentats de Madrid, cette façon de formuler les choses est souvent circonscrite à travers un discours légaliste invoquant la notion de punition. Ce n'est pas la mort qui est exigée en tant que telle, mais la peine de mort : "Pena de muerte para los terroristas, esto es una guerra, ellos o nosotros, basta ya de tolerancia » [La peine de mort pour les terroristes, c'est une guerre, eux ou nous, ça suffit la tolérance] (Archivo del Duelo, EP, Atocha, 17 juin 2004); «SOLUCION POR ELLOS / PENA DE MUERTE» [Solution pour eux / peine de mort] (Archivo del Duelo, DP-0470b).

Dans les messages collectés par les Archives de Paris après les attentats de 2015, nous n'avons pu repérer qu'un seul message rejetant explicitement l'acte de vengeance en tant que tel : «Il ne faut pas pleurer, ni se venger» (Archives de Paris, 3904Woo5_070[01]). Cependant, nous avons remarqué quelques messages qui rejettent la vengeance en tant qu'elle s'inscrit dans une spirale de la violence : «bannirons toute vengeance cruelle» (Archives de Paris, 3910W3[129]) ; «Non à la vengeance sans fin! » (Archives de Paris, fonds privé, AD075HO_027FI_08_001_0913) ; «Sortir du Labyrinthe de la vengeance sans fin» (Archives de Paris, fonds privé, AD075HO_027Fl_20_002_0047). À Madrid, en revanche, deux tiers des 80 messages numériques dans lesquels apparaissent les termes vengar (venger) ou venganza (vengeance) rejettent ce concept : "Si para vengar la muerte de unos, matamos a otros, que sentido tiene?» [Si pour venger la mort de certains, nous en tuons d'autres, quel sens cela a-t-il ?] (Archivo del Duelo, EP, Atocha, 21 septembre 2004); "Que triunfe el amoryno las venganzas» [Que l'amour triomphe et non la vengeance] (Archivo del Duelo, EP, Atocha, 29 juin 2004) ; «Lucharemos, no porvenganza, sino por la paz» [Nous nous battrons, non pour la vengeance, mais pour la paix] (Archivo del Duelo, EP, Atocha, 4 janvier 2005).

Par ailleurs, force est de constater qu'un certain nombre de redéfinitions de la notion de vengeance provoquent un glissement de sens en refonctionnalisant des actes et notions reconnus par la société. Font ainsi fonction d'acte vengeur :

1) L'acte d'écriture : «Ces messages [...] se transforment en balles» (Archives de Paris, 3904W43[3]); "J'écris ces phrases assassines pour meurtrir leurs actions sanglantes avec ces cons sonnes» (Archives de Paris, 3904W19[5]); 
2) Le pardon: «Sachez [...] qu'il n'est pas de plus grande vengeance que de donner son Pardon, mais ça, vous ne pouvez pas le comprendre...» (Archives de Paris, 3910W1[11]);

3) La joie de vivre : "VIVONS NOS VALEURS. C'EST LA PLUS BELLE REVANCHE» (Archives de Paris, 3907W3[21]); "La mejor venganza es la alegria de VIVIR! » [La meilleure vengeance est la joie de vivre!] (Archivo del Duelo, EP, Atocha, 20 octobre 2004);

4) La paix, l'amour et la solidarité : «Nuestra mejor venganza... el amor y la solidaridad» [Notre meilleure vengeance... l'amour et la solidarité] (Archivo del Duelo, EP, mascercanos.com, 10 juin 2004); «Nuestra venganza sera la paz» [Notre revanche sera la paix] (Archivo del Duelo, EP, mascercanos.com, 11 octobre 2004); "queremos respirar paz, esa debe ser la venganza» [nous voulons respirer la paix, cela doit être la vengeance] (Archivo del Duelo, EP, mascercanos.com, 11 octobre 2004);

5) La société démocratique : "La mejor venganza es seguir siendo una sociedad democrática y no sexista» [La meilleure revanche est de rester une société démocratique et non sexiste] (Archivo del Duelo, EP, Atocha, 9 janvier 2005).

De telles redéfinitions constituent une ressource rhétorique importante en tant qu'elles permettent de briser la dichotomie entre vouloir-faire (désir de vengeance) et faire (acte de vengeance). Dans l'exemple nº 1, l'acte d'écriture luimême équivaut à un acte vengeur. Ainsi le sujet de l'énonciation s'appropriet-il une sorte d'agency en exécutant l'acte vengeur lui-même à travers l'écriture de son message. L'exemple $n^{\circ} 2$, lui, assimile le pardon à un acte vengeur. Or, tandis que pardonner est un acte de langage performatif, se venger ne l'est pas. L'assimilation du pardon à l'acte de vengeance change par conséquent la direction d'ajustement (direction offit) au sens de John R. Searle (1985). Au lieu de s'engager à changer le monde par un futur acte de vengeance, le monde est changé immédiatement par l'acte de langage performatif, exempt de violence physique. Les autres exemples réinterprètent la pratique des valeurs occidentales comme acte de vengeance, supposant que les terroristes ne supportent pas que leurs actes cruels n'aient pas l'effet souhaité, à savoir déstabiliser la société frappée et ses valeurs. Évidemment, si la pratique des valeurs occidentales est mise au même rang que l'acte de vengeance, les énonciateurs s'approprient une agency qu'ils ne posséderaient pas si l'acte de vengeance impliquait des actions violentes dirigées contre les responsables de l'attentat.

De fait, un message de vengeance ne peut plus être adressé aux responsables directs de l'acte terroriste, si ceux-ci, comme dans le cas des attentats de Paris, ont été tués par les forces spéciales au cours de leur attaque ou peu après ${ }^{17}$. Il est dès lors intéressant d'examiner comment les énonciateurs pallient cette

17. Dans le cas de Madrid, en revanche, la situation est plus complexe. Le 3 avril 2004, juste avant l'assaut de la police espagnole, sept terroristes se sont fait exploser dans leur appartement de Leganés, dans la banlieue de Madrid. 28 personnes ont été traduites en justice en 2006. 21 ont été reconnues coupables et sept acquittées. 
difficulté en attribuant le rôle sémantique du patient à des destinataires alternatifs. Les observations menées sur les messages vengeurs provenant du corpus de Paris montrent que ce rôle est en premier lieu attribué à Daech, qui a revendiqué la responsabilité des attentats. Nous n'avons en revanche rencontré que très peu de messages au contenu raciste ou islamophobe, qui viseraient «les musulmans » ou « les Arabes » en général - constat qui rejoint celui déjà dressé par C. Sánchez-Carretero (2011b, p. 257) et G. Truc (2016, p. 250) concernant les messages issus des mémoriaux populaires de Madrid.

Nous avons observé que le sujet de l'énonciation ne prend que rarement le rôle sémantique d'agent, en raison probablement de la responsabilité liée à l'appropriation de l'agency. Dans cette perspective, nous avons identifié différentes stratégies rhétoriques, qui permettent d'assumer la responsabilité de l'action vengeresse à des degrés divers. Dans les deux corpus, nous avons repéré quelques messages tentant d'occulter l'agent par l'emploi du passif18 - par exemple : «Vous serez venger» (Archives de Paris, 3904W67[112]) - ou de constructions impersonnelles. D’autres moyens linguistiques employés à cette fin sont par exemple l'expression d'un simple désir que la vengeance soit perpétrée ("J'espèrent qu’il vont le régréter en enfer», Archives de Paris, 3904W5[214]) ou l'usage de l'impératif ("Allez, crevez!", Archives de Paris, 3904W45[185]). Dans le corpus espagnol, nous avons aussi trouvé des constructions impersonnelles et passives intégrées dans les actes de langage comme le souhait - «Espero que la venganza sea terrible» [J'espère que la vengeance sera terrible] (Archivo del Duelo, EP, mascercanos.com, 23 août 2004) -, ou la demande - "Solo pido venganza, y eso algun dia se conseguirá» [Je ne demande que la vengeance, et un jour cela sera accompli] (Archivo del Duelo, $\mathrm{EP}$, Atocha, 8 janvier 2005). Le message suivant formule une menace indirecte en recourant au verbe pagar (payer) : «Los que os hicieron esto algún día lo pagarán muy caro » [Ceux qui vous ont fait cela le paieront très cher un jour] (Archivo del Duelo, EP, Atocha, 15 juin 2004). Cependant, la plupart des messages de vengeance tentent de réaliser le rôle sémantique d'agent, bien que de manière différente. Au lieu de l'agent prototypique - l'être humain qui agit et contrôle son action -, d'autres référents sont mis en jeu :

Un Dieu vengeur - Dans quelques messages, le rôle de l'agent se voit conféré à Dieu : "Si quelqu'un détruit le temple de Dieu, Dieu le détruira. Car le temple de Dieu est saint et ce temple c'est vous (Saint Paul, 1 Co 3,16)» (Archives de Paris, 3904W7[59]) ; "L’Éternel [...] déteste le méchant et celui qui aime la violence / II fait pleuvoir sur les méchants des charbons, du feu et du soufre [...] (Psaume XI)» (Archives de Paris, 3904W55[107]) ; «Que Dios castigue a quienes hicieron sufrir a tantas familias inocentes» [Que Dieu punisse ceux qui ont fait souffrir tant de familles innocentes] (Archivo del Duelo, EP, Ato-

18. Cette stratégie a été étudiée par Alessandro Duranti (2004). 


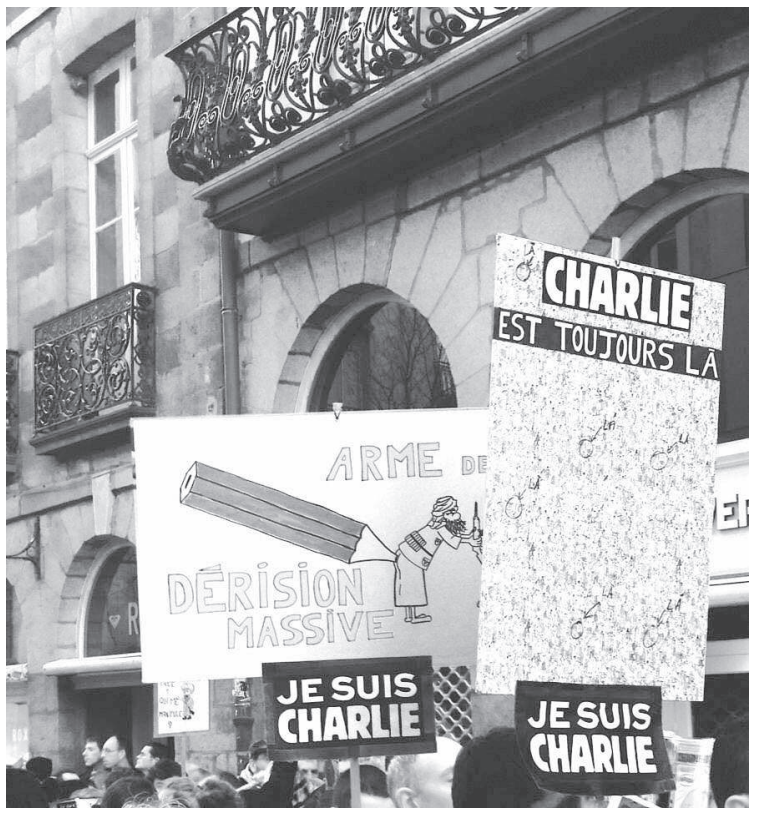

Figure 1. Photographie prise lors de la marche républicaine le 11 janvier 2015 à Rennes

(c) Tatiana Châtelet. Ce dessin fait également partie du corpus mis en ligne par les Archives de Rennes, fonds numérique "Mémoires des attentats / Janvier 2015", image 45 sur 103.

cha, 14 octobre 2004). La foi chrétienne dénie aux hommes la capacité d'agir effective relative à la vengeance, celle-ci étant l'apanage de Dieu (Rom. 12, 19; Dt. 32, 35). L'attribution d'agency à un être métaphysique tel que Dieu, reconnu comme moralement supérieur, permet d'exprimer le désir de vengeance sans devoir pour autant rompre avec les conventions sociales et morales. Compte tenu de l'importance que revêt en France la laïcité, un tel recours à la référence religieuse apparait tout à fait remarquable, quand bien même il reste marginal.

Les forces naturelles - De rares messages du corpus parisien modulent l'agency en accordant la capacité d'agir aux forces naturelles : «Un éclair surnaturel jaillira, suivi d'un grondement de tonnerre qui roulera interminablement sur les deux villes du Califat "l'apocalypse" pour faire disparaître DAECH » (Archives de Paris, 3904W7[62]). Dans ce contexte, l'adjectif descriptif surnaturel en relation avec l'apocalypse constitue une référence implicite à la colère de Dieu. Dans le corpus espagnol, on en trouve aussi un exemple, où l'image de l'éclair est également présente, mais désigne, par métaphore, les yeux des victimes qui punissent les agresseurs : "Que los ojos de las víctimas sean relámpagos en las noches de sus asesinos» [Que les yeux des victimes soient des éclairs dans les nuits de leurs meurtriers] (Archivo del Duelo, EP, mascercanos.com, 18 juin 2004).

Des artefacts (mythiques) - Dans les messages collectés à Paris, nous avons isolé quelques documents modulant l'agency principalement par le truchement d'éléments visuels. Dans la figure 1 , le crayon est détourné de sa 
fonction et utilisé comme une arme. En tant qu'objet inanimé, le crayon est porteur d'une charge mythique et iconique importante. Après les attentats contre Charlie Hebdo, celui-ci est devenu un symbole ayant servi à désigner, par métonymie, la liberté de la presse (Bazin, 2016). La valeur connotative du crayon est reprise dans le message contextualisant le dessin. D'un point de vue lexical, il joue sur l'allitération destruction/dérision en opposant les « armes de dérision massive » aux «armes de destruction massive ». Doté d'une capacité d'action effective, le crayon est construit en tant qu'agent de l'acte vengeur, qui peut alors se réaliser sans confier de responsabilité à un être humain. Nous n'avons pas trouvé d'exemples comparables dans le corpus espagnol.

La ville de Paris (en tant qu'entité mythique) - Dans le corpus parisien se trouvent plusieurs messages tant textuels - «Paris va se venger» (Archives de Paris, 3904W49[74]) - que visuels (figures 2 et 3) attribuant l'agency à la ville de Paris elle-même. «Paris » peut ici être compris de différentes manières : comme le lieu des attentats et ainsi, à travers une personnification, comme la victime directe de ceux-ci, comme une entité spatiale construite par les habitants, ou bien comme l'ensemble des Parisiens. Dans cette perspective, la tour Eiffel (figures 2 et 3) semble condenser Paris sous la forme d'une synecdoque. C'est la tour Eiffel qui accomplit la contre-attaque en poignardant ou en écrasant les terroristes. Étant donné la charge mythique liée à la tour Eiffel (Barthes, 1964b), elle suscite l'imagination, n'étant pas seulement un monument touristique, mais aussi un symbole de la modernité, des valeurs françaises et de la Ville Lumière en tant que telle. La contextualisation par le hashtag \#NOTAFRAID souligne la détermination de Paris à contre-attaquer. Une telle présentation permet la réalisation visuelle des actes de vengeance causant la mort des terroristes, sans cependant attribuer de responsabilité à la société civile en tant que telle.

La figure 3 combine ainsi trois mythes différents. En faisant de la tour Eiffel le talon d'un escarpin bleu, blanc, rouge, le mythe de la tour Eiffel se trouve relié à celui de Paris, capitale de la mode, évoquant par association l'élégance, le luxe et la séduction. Dans cette optique, la représentation visuelle de la pointe de la tour Eiffel déclenche une association d'idées avec l'embout d'un crayon, porteur également d'une valeur symbolique puisqu'il renvoie à la liberté d'expression et ainsi, implicitement, à l'attentat de janvier.

Là encore, nous n'avons pas trouvé d'équivalents dans les données espagnoles. Dans le corpus numérique apparaissent en revanche plusieurs références à la mythification populaire de Madrid al cielo (de Madrid au paradis) - slogan qui évoque la splendeur de la capitale espagnole, qui n'est surpassée que par le paradis. Étant donné la nuance de sens macabre que les attentats y ajoutent, la prédominance de ce slogan dans les données espagnoles est surprenante. Un seul message mentionne : "De Madrid al cielo, pero nunca mas de esta manera» [De Madrid au paradis, mais plus jamais de cette façon] (Archivo del Duelo, EP, mascercanos.com, 16 juin 2004). Dans de nombreux 


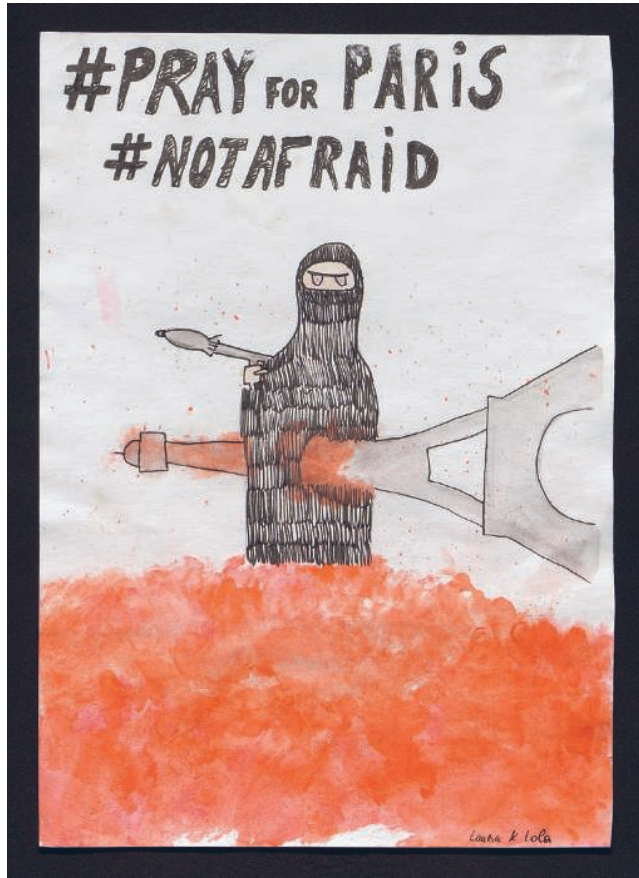

Figure 2. Archives de Paris, 3904W10(51), 2015

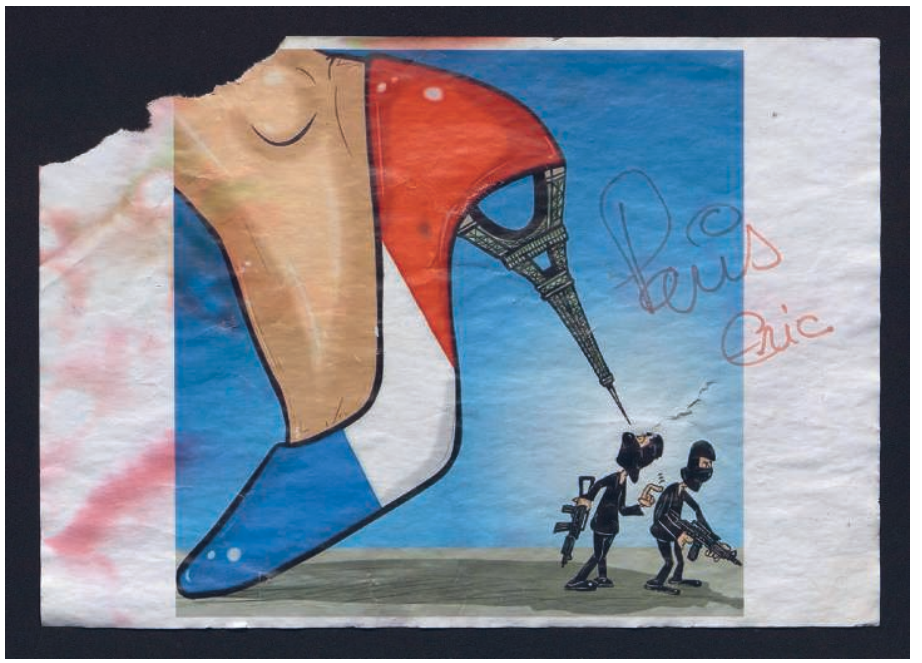

Figure 3. Archives de Paris, 3904W10(15), 2015 
autres exemples, l'évocation du mythe de Madrid signifie plutôt un geste de résilience : "Madrid Resiste, Madrid Vive, Ahora más que nunca, de Madrid al cielo" [Madrid résiste, Madrid vit, plus que jamais, de Madrid au paradis] (Archivo del Duelo, EP, mascercanos.com, 10 juin 2004). Ce geste de résilience, on l'a vu, peut d'ailleurs être associé à la vengeance, comme dans «La mejor venganza es la alegria de VIVIR! » [La meilleure vengeance est la joie de vivre!] (Archivo del Duelo, EP, Atocha, 20 octobre 2004), la joie de vivre renvoyant à une représentation mythique de la culture occidentale, constituée ici en arme infligeant des souffrances aux islamistes qui la refusent.

Un «nous » collectif - Une autre façon de réduire la responsabilité consiste à recourir à un «nous » collectif désignant un groupe de personnes indéfini dans lequel s'inclut le sujet de l'énonciation : « Nous allons vous venger » (Archives de Paris, 3904W8[132_01]) ; "ojo porojo y diente por diente vengaremos vuestra muerte » [œil pour œil et dent pour dent nous vengerons votre mort] (Archivo del Duelo, EP, Atocha, 9 octobre 2004). L'individu ne s'attribue de l'agency subjective qu'au sein d'un collectif. Conformément à l'analyse de G. Truc (2016), différentes échelles du «nous» se laissent ici distinguer. Dans les données françaises, on rencontre notamment des termes désignant des collectivités au niveau national ("Nous peuple de France », Archives de Paris, 3905W1[2]) ainsi que des collectifs politiques dans lesquels le sujet de l'énonciation s'inclut de manière implicite : "La République aura ta peau (Archives de Paris, 3909W2[28]) ; «la France traquera jusqu'au dernier terroriste» (Archives de Paris, 3904W34[16]). Nous n'avons pas trouvé d'exemples comparables dans le corpus de Madrid. En revanche, nous avons remarqué la construction imaginaire d'un collectif vengeur, qui inclut un «nous» indéterminé ainsi que le groupe des victimes : "juntos nosotros y los que se han ido podremos hacer justicia para vengarnos de esta gente» [ensemble, nous et ceux qui s'en sont allés pourrons faire justice pour nous venger de ces gens] (Archivo del Duelo, EP, Atocha, 30 décembre 2004).

Le sujet de l'énonciation - Enfin, nous n'avons repéré que peu d'exemples attestant une attribution d'agency immodérée au sujet de l'énonciation luimême. C'est souvent la relation directe à l'une des victimes qui peut susciter un sentiment de responsabilité et de devoir se manifestant dans le désir d'assumer personnellement le rôle du vengeur : «Je veux les venger, Je veux venger Mon Amie Marion partie trop tôt» (Archives de Paris, 3904W4[4]). Parmi les messages numériques du corpus madrilène, nous n'avons trouvé qu'un seul exemple dans lequel le sujet de l'énonciation assume l'entière responsabilité de l'action vengeresse : «LA VENGANZA ESTÁ SERVIDA, NO DESCANSO HASTA ENCONTRARLOS» [La vengeance est servie, pas de repos tant que je ne les aurai pas trouvés] (Archivo del Duelo, EP, Atocha, 7 janvier 2005). Dans un autre exemple, la responsabilité se limite au désir de vengeance : "siento odio y ganas de vengarme» [je ressens de la haine et l'envie de me venger] (Archivo 
del Duelo, EP, Atocha, 28 novembre 2004). Un troisième exemple reste ambigu entre une lecture qui l'interprète comme l'expression d'un sentiment ou bien comme la description d'un potentiel d'action croissant : «En mi, crece venganza» [En moi, grandit la vengeance] (Archivo del Duelo, EP, mascercanos. com, 22 septembre 2004).

Dans les messages parisiens, nous avons par ailleurs repéré deux messages modulant l'agency personnelle à travers la projection dans un espace imaginaire :

Je te dirais que puisque tu aimes la mort, tire-toi une balle, va rejoindre ton avatar de Dieu et laisse nous en rire. (Archives de Paris, 3904W13[17])

Je ferais mieux de jeter mon dictionnaire. Et de m'acheter une Kalachnikov et des grenades, pour m'en aller distribuer à mon tour amour et compassion partout où vous vous trouvez. Avant de laisser, sur vos corps enfin bénis, la photo de ma cousine Madeleine, que votre miséricorde a lâchement assassinée vendredi au Bataclan. (Archives de Paris, 3904W54[75])

Sur le plan grammatical, l'ouverture de ces espaces imaginaires est marquée par l'emploi du conditionnel (je ferais mieux; je te dirais). Au sein de telles projections, le sujet de l'énonciation peut céder à son désir de vengeance (vouloir-faire) et assumer le rôle de vengeur en mettant en scène l'acte vengeur. Le deuxième exemple met en lumière une relation personnelle à une des victimes (ma cousine), ce qui conforte notre hypothèse selon laquelle la réclamation d'agency par le sujet de l'énonciation est réservée aux proches des victimes. A contrario, dans le premier exemple, à part l'attribution d'agencyau je, un nous indéfini est également agentivé en raillant le terroriste auquel le message est adressé (tu).

Nous supposions au début de cet article qu'en l'absence d'une capacité d'agir effective, le langage représente un instrument compensateur en mettant à la disposition de l'énonciateur des ressources linguistiques qui lui permettent d'exprimer son désir de vengeance d'une manière socialement acceptable. Dans cette optique, le langage de la vengeance n'est pas sans importance psychologique dans le processus de deuil. L'atténuation rhétorique de la responsabilité de l'énonciateur s'est révélée être un aspect déterminant. Les énonciateurs assignent à très peu de sujets de l'énonciation l'entière responsabilité d'une action vengeresse, ne serait-ce qu'imaginée. Ayant à disposition une multitude de possibilités linguistiques (au niveau sémantique, syntaxique et lexical), les énonciateurs adoptent celles (actes de langages, redéfinitions, projections) qui leur permettent d'éviter les conflits moraux en modulant l'agency de façon à ventiler la responsabilité de l'action : ils délèguent le rôle sémantique de l'agent à un groupe (indéfini), des forces (sur-)naturelles ou bien des artefacts. Les mythes et valeurs de la société occidentale sont aussi mis à profit. 
Mais c'est surtout après les attentats de Paris que la charge symbolique des mythes apparait la plus exploitée, davantage qu'après ceux de Madrid. Même si la fonction de catharsis constitue un élément fort du langage de la vengeance, celle-ci rencontre aussi ses limites. Au niveau du discours, la charge modale contradictoire (et notamment l'impératif de respect des valeurs sociales) s'oppose à la réalisation de tout le potentiel linguistique. Au niveau des actes de langage, les circonstances liées à l'attentat considéré (mort des responsables directs) peuvent limiter la traduction langagière de la notion de réciprocité (sous sa forme œil pour œil, dent pour dent) à une stratégie de projection, permettant de confronter directement coupable et vengeur dans un espace imaginaire. Mais celle-ci ne semble employée que rarement. En comparant les corpus français et espagnol, nous avons enfin pu noter des différences importantes. Ainsi, dans les messages de réaction à l'attentat de Madrid, figurent en fin de compte très peu de messages évoquant le désir de se venger des terroristes, ce qui pourrait s'expliquer par le fait que le gouvernement conservateur dirigé par José María Aznar fut considéré comme responsable des attentats en raison de son engagement dans la guerre en Irak, peu populaire à l'époque en Espagne. En outre, les messages de vengeance sont pour la plupart, en comparaison avec ceux rédigés à la suite des attentats de Paris, moins élaborés et plus courts. Dans le corpus espagnol, nous n'avons par exemple trouvé aucun recours à des moyens d'expression visuelle de la vengeance. Notre recherche est encore en cours. Dans le cadre d'un examen plus vaste, il faudrait, à l'évidence, discuter en détail des conditions de production des messages, et des différences en la matière entre des textes élaborés, voire littéraires, conçus et rédigés chez soi, et des messages griffonnés à la hâte, place de la République à Paris, ou dans la gare d'Atocha à Madrid. Nous pensons cependant avoir jeté ici les bases d'une nécessaire étude du langage de la vengeance dans les réactions à des attentats, en tant que celui-ci participe de l'émotion populaire et du deuil collectif que ces événements produisent.

\section{Références}

Austin John L., 1975, How to Do Things with Words, J. O. Urmson et M. Sbisà éd., Cambridge, Harvard University Press.

BARThes Roland, 2014 [1957], Mythologies, Paris, Seuil.

- 1964a, «Rhétorique de l'image», Communications, nº 4, p. 40-51.

- 1964b, La Tour Eiffel, Paris, Delpire.

BAzıN Maëlle, 2016, "Brandir un crayon : geste-emblème des rassemblements postattentats de janvier 2015", Mots. Les langages du politique, nº110, p. 67-81.

Bethmann Stephanie, Helfferich Cornelia, Hoffmann Heiko et al. éd., 2012, Agency: Qualitative Rekonstruktionen und gesellschaftstheoretische Bezüge von Handlungsmächtigkeit, Weinheim, Beltz Juventa. 
BoltANSKI Luc, 1990, L'amour et la justice comme compétences : trois essais de sociologie de l'action, Paris, Métailié.

Connolly William E., 2005, Pluralism, Durham, Londres, Duke University Press.

Doss Erika, 2006, "Spontaneous memorials and contemporary modes of mourning in America », Material Religion, vol. II, n³ 3, p. 294-319.

DURANTI Alessandro, 2004, "Agency in language », dans A Companion to Linguistic Anthropology, A. Duranti éd., Malden, Oxford, Victoria, Blackwell, p. 451-474.

Elshout Maartje, Nelissen Rob M. A., VAN BEest Ilja, 2015, «A prototype analysis of vengeance », Personal Relationships, vol.XXII, n³ 3, p. 502-523.

ELSTER Jon, 1990, «Norms of revenge », Ethics, vol. C, nº 4, p. 862-885.

ERMAN Michel, 2012, Éloge de la vengeance : essai sur le juste et la justice, Paris, PUF.

FRAENKEL Béatrice, 2011, "Street shrines and the writing of disaster: 9/11, New York, 2001 », dans Grassroots Memorials: The Politics of Memoralizing Traumatic Death, P. J. Margry et C. Sánchez-Carretero éd., New York, Oxford, Berghahn Books, p. 229-243.

- 2007, «Actes d'écritures : quand écrire c'est faire », Langage et société, n 121-122, p. 101-112.

GenSBURGER Sarah, 2017, Mémoire vive : chroniques d'un quartier, Bataclan 2015-2016, Paris, Anamosa.

GREIMAS Algirdas Julien, 1976, «Pour une théorie des modalités », Langages, n 43 , p. 90-107.

Greimas Algirdas Julien, FontANILLE Jacques, 1991, Sémiotique des passions : des états de choses aux états d'âme, Paris, Seuil.

HELFFERICH Cornelia, 2012, «Einleitung: Von roten Heringen, Gräben und Brücken. Versuch einer Kartierung von Agency-Konzepten », dans Agency: Qualitative Rekonstruktionen und gesellschaftstheoretische Bezüge von Handlungsmächtigkeit, S. Bethmann et al. éd., Weinheim, Beltz Juventa, p. 9-40.

HÉnAfF Marcel, 2000, "La dette de sang et l'exigence de justice», dans Comprendre pour agir : violences, victimes et vengeances, P. Dumouchel et al. éd., Paris, Laval, L'Harmattan, Presses universitaires de Laval, p. 31-64.

HJeLmSLev Louis, 1961 [1943], Prolegomena to a Theory of Language, F. J. Whitfield trad., Madison, University of Wisconsin Press.

KaIluWeit Rolf, Hummel Martin éd., 2004, Semantische Rollen, Tübingen, Narr.

LUCIUS-HoEnE Gabriele, 2012, “ „Und dann haben wir’s operiert”. Ebenen der Textanalyse narrativer Agency-Konstruktionen », dans Agency: Qualitative Rekonstruktionen und gesellschaftstheoretische Bezüge von Handlungsmächtigkeit, S. Bethmann et al. éd., Weinheim, Beltz Juventa, p. 40-71.

MARgry Peter Jan, SÁnchez-CARRETERo Cristina éd., 2011a, Grassroots Memorials: The Politics of Memoralizing Traumatic Death, New York, Oxford, Berghahn Books.

- 2011b, «Rethinking memorialization: the concept of grassroots memorials», dans Grassroots Memorials: The Politics of Memoralizing Traumatic Death, P. J. Margry et C. Sánchez-Carretero éd., New York, Oxford, Berghahn Books, p. 1-51.

McCullough Michael E., 2008, Beyond Revenge: The Evolution of the Forgiveness Instinct, San Francisco, Jossey-Bass.

ORTIz GARCíA Carmen, 2013, "Pictures that save, pictures that soothe: photographs at the grassroots memorials to the victims of the March 11, 2004 Madrid bombings », Visual Anthropology Review, vol.XXIX, nº 1, p. 57-71.

- 2011, "Memoriales del atentado del 11 de marzo de Madrid», dans El Archivo del Duelo: análisis de la respuesta ciudadana ante los atentados del 11 de marzo en 
Madrid, C. Sánchez-Carretero éd., Madrid, CSIC, p. 33-65.

QUINTANA Aldina, KaILUWEIT Rolf, 2017, «Intensificadores de un lenguaje de duelo: el Espacio de palabras de Atocha (2004-2005) del 11-M», dans Atenuación e intensificación en diferentes géneros discursivos, M. Albelda Marco et W. Mihatsch éd., Madrid, Iberoamericana Vervuert, p. 221-245.

RAPPAPORT Hovav Malka, LEVIn Beth, 2015, "The syntax-semantics interface: semantic roles and syntactic arguments ", dans The Handbook of Contemporary Semantic Theory, S. Lappin et C. Fox éd., Oxford, Blackwell, p. 593-634.

ReINARES Fernando, 2014, ¡Matadlos! Quién estuvo detrás del 11-M y por qué se atentó en España, Madrid, Galaxia Gutenberg.

Rosenbaum Thane, 2013, Payback: The Case for Revenge, Chicago, Londres, The University of Chicago Press.

SÁnCHEZ-CARRETERo Cristina éd., 2011a, El Archivo del Duelo: análisis de la respuesta ciudadana ante los atentados del 11 de marzo en Madrid, Madrid, CSIC.

- 2011b, "The Madrid train bombings: enacting the emotional body at the March 11 grassroots memorials ", dans Grassroots Memorials: The Politics of Memoralizing Traumatic Death, P. J. Margry et C. Sánchez-Carretero éd., New York, Oxford, Berghahn Books, p. 244-262.

SANTINO Jack, 2011, «Between commemoration and social activism: spontaneous shrines, grassroots memorialization, and the public ritualesque in Derry », dans Grassroots Memorials: The Politics of Memoralizing Traumatic Death, P. J. Margry et C. SánchezCarretero éd., New York, Oxford, Berghahn Books, p. 97-108.

SEARLE John R., 1985, L'intentionalité: essai de philosophie des états mentaux, C. Pichevin trad., Paris, Minuit.

Steen Pamela, 2012, «Rekonstruierte, imaginierte und performative Agency in der verbalen Interaktion », dans Agency: Qualitative Rekonstruktionen und gesellschaftstheoretische Bezüge von Handlungsmächtigkeit, S. Bethmann etal. éd., Weinheim, Beltz Juventa, p. 238-269.

TRUC Gérôme, 2016, Sidérations : une sociologie des attentats, Paris, PUF.

- 2011, "Espacio de Palabras y rituales de solidaridad en Atocha», dans El Archivo del Duelo: análisis de la respuesta ciudadana ante los atentados del 11 de marzo en Madrid, C. Sánchez-Carretero éd., Madrid, CSIC, p. 207-226.

- 2006, «Le cosmopolitisme sous le coup de l'émotion : une lecture sociologique des messages de solidarité en réaction aux attentats du 11 mars 2004 à Madrid », Hermès, $n^{0} 46$, p. 189-199.

TRUc Gérôme, BAzın Maëlle, 2019 (à paraître), «Les gardiens de la mémoire : mobilisations et conflits d'appropriation autour de mémoriaux post-attentats à Madrid, Londres et Paris », Ethnologie française.

\section{Résumé / Abstract / Compendio}

\section{Ressources et limites d'un langage de la vengeance dans les réactions popu- laires aux attentats de Madrid (2004) et Paris (2015)}

Notre contribution propose une approche sémiolinguistique qui vise à mettre en perspective les enjeux soulevés par l'émergence d'un langage de la vengeance en réponse aux attaques terroristes. À partir d'une étude comparative des réactions civiles aux 
attentats de Madrid en 2004 et de Paris en 2015, elle cherche à appréhender les stratégies linguistiques (actes de langage, redéfinitions, projections, imaginations) mises en œuvre pour exprimer le désir de vengeance, bien que la vengeance soit en général socialement considérée comme inappropriée. Dans cette optique, l'accent est mis sur le rôle sémantique de l'agent en explorant la construction de l'agency (capacité d'agir effective ou imaginée) dans la zone conflictuelle entre vouloir-faire (désir de vengeance motivé par la souffrance et la colère) et ne-pas-pouvoir-faire (déterminé par les normes sociales, morales et juridiques).

Mots-clés : attentats terroristes, Madrid 2004, Paris 2015, vengeance, langage, agency

\section{Resources and limits of a retaliatory language in the popular reactions to the attacks in Madrid (2004) and Paris (2015)}

Our contribution proposes a semiolinguistic approach that accounts for the issues raised by the emergence of a language of revenge in response to terrorist attacks. Based on a comparative study of civil reactions in 2004 Madrid and 2015 Paris, our approach aims to understand the linguistic strategies (speech acts, redefinitions, projections, imaginations) that are used to express the desire for revenge, in spite of revenge generally being considered socially inappropriate. In this perspective, we focus on the semantic role of the agent by exploring the construction of agency (the real or imagined ability to act) in the conflict zone between wanting to act (a desire for revenge motivated by suffering and anger) and not being able to act (determined by social, moral and legal norms).

Keywords: terrorist attacks, 2004 Madrid, 2015 Paris, revenge, language, agency

\section{Recursos y límites de un lenguaje de venganza en las reacciones populares frente a los atentados de Madrid (2004) y Paris (2015)}

Nuestra contribución propone un enfoque semio-lingüístico que intenta poner de relieve los problemas planteados por el surgimiento de un lenguaje de venganza en respuesta a los ataques terroristas. Basado en un estudio comparativo de las reacciones civiles en Madrid 2004 y París 2015, nuestro objetivo es comprender las estrategias lingüísticas (actos de lenguaje, redefiniciones, proyecciones, imaginarios) utilizadas para expresar el deseo de venganza, aunque la venganza sea, por lo general, considerada socialmente inapropiada. Desde esta perspectiva, se hace hincapié en el rol semántico del agente al explorar la construcción de agency (capacidad de actuar de forma real o imaginaria) en la zona de conflicto entre el vouloir-faire (deseo de venganza motivado por el sufrimiento) y el ne-pas-pouvoir-faire (determinado por las normas sociales, morales y legales).

Palabras claves: ataques terroristas, Madrid 2004, París 2015, venganza, lenguaje, agency 